\title{
Regularity of critical invariant circles of the standard nontwist map
}

\author{
A Apte ${ }^{1,4}$, Rafael de la Llave ${ }^{2}$ and Nikola P Petrov ${ }^{3}$ \\ ${ }^{1}$ Department of Physics, University of Texas at Austin, 1 University Station C1500, Austin \\ TX 78712-0262, USA \\ 2 Department of Mathematics, The University of Texas at Austin, 1 University Station C1200, \\ Austin, TX 78712-0257, USA \\ ${ }^{3}$ Department of Mathematics and Michigan Center for Theoretical Physics, University of \\ Michigan, Ann Arbor, MI 48109-1109, USA \\ E-mail: apte@physics.utexas.edu, 1lave@math.utexas.edu and npetrov@umich.edu
}

Received 10 October 2004, in final form 20 January 2005

Published 16 February 2005

Online at stacks.iop.org/Non/18/1173

Recommended by A Chenciner

\begin{abstract}
We study critical invariant circles of several noble rotation numbers at the edge of break-up for an area-preserving map of the cylinder, which violates the twist condition.

These circles admit essentially unique parametrizations by rotational coordinates. We present a high accuracy computation of about $10^{7}$ Fourier coefficients. This allows us to compute the regularity of the conjugating maps and to show that, to the extent of numerical precision, it only depends on the tail of the continued fraction expansion.
\end{abstract}

PACS numbers: 05.10.Cc, 02.30.Nw, 02.30.-f, 47.53.+n

\section{Introduction}

Area-preserving maps of a cylinder have been studied for several decades, both as lowdimensional models of physical systems and as interesting mathematical problems. Until recently, most of these studies have dealt with twist maps, which are the maps that satisfy a certain non-degeneracy condition. Lately, maps that violate this condition locally in phase space have been of great interest. These nontwist maps occur in various physical systems such as, e.g., in the study of magnetic field lines in toroidal plasma devices with reversed shear profile, channel flows, and other physical applications (see, e.g. [1-8]).

${ }^{4}$ Current address: Department of Mathematics, University of Carolina, CB 3250 Phillips Hall, Chapel Hill, NC 27599, USA.E-mail: apte@email.unc.edu 
Nontwist maps present new phenomena that are not observed in twist maps. Some of these phenomena (reconnection, bifurcation, meandering tori, etc) are discussed in [8-14]. It should also be remarked that nontwist maps appear naturally in iterations of twist maps. This is because of the fact that, even though the twist property has dynamical consequences, it is not preserved by composition or by a change of variables.

These maps are also of mathematical interest because many important results about maps, e.g., KAM and Aubry-Mather theory, depend on the twist condition. Even though Aubry-Mather variational theory for twist maps does not generalize for nontwist maps, there is an analogue of KAM theory. In $[15,16]$ it was suggested that one can continue shearless circles with a Diophantine rotation number provided that one adjusts a second parameter; this was proved rigorously in [17]. Furthermore, in [15,16] it was observed numerically that if the curve is continued, one reaches a critical value where the circles cannot be continued anymore. The circle that cannot be continued-henceforth referred to as the critical circle (or circle at break-up) - enjoys remarkable self-similar properties, which are universal (i.e. they do not seem to depend on the map). The calculations of critical shearless invariant tori in [16] were extended and refined in [18-21].

As we will show later, a natural way of representing the circle is by giving some parametrization in which the motion becomes a rotation. This parametrization is essentially unique. The study of the Fourier spectrum reveals several properties of the function. One such property is the regularity of the parametrization. The study of the regularity of functionswhich is crucial in KAM theory-has been studied extensively in the mathematical literature. Efficient algorithms for problems very similar to those considered here were developed in [22].

In this paper, we analyse further the properties of the critical invariant tori with noble winding numbers computed in $[18,19,21]$ by using techniques developed in [23]. Since the new calculations in $[18,19,21]$ allow us to compute accurately orbits of length of several millions, we have a very accurate computation of the critical invariant circle.

Remark 1. The standard nontwist maps present several symmetries that makes the calculation simpler. On the other hand, it is expected from the renormalization group picture that the regularities of the shearless circles of other nontwist maps without these symmetries should also be the same as found here.

Remark 2. The regularities for shearless circles with non-noble winding numbers are not considered here. Indeed, the breakdown of shearless circles with non-noble numbers has not been considered in the literature and it is an open question whether there is a renormalization group description for them.

We have found that the functions corresponding to the critical shearless circle saturate several bounds in harmonic analysis, so that the regularity is easy to ascertain. In the cases we have examined, the regularity of the critical circles is the same for different rotation numbers with the same tail of the continued fraction expansions. This is consistent with the predictions of universality that follow from the renormalization group picture [24].

This paper is organized as follows. Section 2 introduces the basic terminology for nontwist maps. In section 3, we describe the convenient parametrization used to study these regularities and its symmetry properties. The numerical techniques are briefly explained in section 4 . We discuss the results in section 5.

\section{Preliminaries}

The standard nontwist map $F: \mathbb{T}^{1} \times \mathbb{R} \rightarrow \mathbb{T}^{1} \times \mathbb{R}$ is given by

$$
y^{\prime}=y-b \sin (2 \pi x), \quad x^{\prime}=x+a\left(1-y^{\prime 2}\right) \bmod 1,
$$


where $(x, y) \in \mathbb{T}^{1} \times \mathbb{R}$ and $a, b$ are parameters. This map violates the twist condition

$$
\frac{\partial x^{\prime}}{\partial y} \neq 0
$$

along the curve $y=b \sin (2 \pi x)$ in phase space. An orbit of the map is the set of points $\left\{\left(x_{i}, y_{i}\right)\right\}_{i=-\infty}^{\infty}$ such that $\left(x_{i+1}, y_{i+1}\right)=F\left(x_{i}, y_{i}\right)$. An orbit is periodic of least period $n$ if $x_{i+n}=x_{i}, y_{i+n}=y_{i}$, and $n$ is the smallest such integer. We also consider the lift of the standard nontwist map $\tilde{F}: \mathbb{R} \times \mathbb{R} \rightarrow \mathbb{R} \times \mathbb{R}$ given by (1) by removing the mod 1 . We denote by $\left\{\left(\tilde{x}_{i}, \tilde{y}_{i}\right)\right\}$ an orbit of the lift map corresponding to the orbit $\left\{\left(x_{i}, y_{i}\right)\right\}$ of the map. The winding (or rotation) number $\omega$ of an orbit is defined as

$$
\omega=\lim _{i \rightarrow \infty} \frac{\tilde{x}_{i}}{i}
$$

if the limit exists. This number is independent of the lift taken. The winding number of a periodic orbit is rational; an orbit for which the winding number does not exist is chaotic. A set homeomorphic to a circle that is mapped to itself by $F$ is called an invariant circle. It was already proved by Poincare that all the points in a circle have the same winding number. The winding number of orbits belonging to these circles is generically irrational.

Invariant circles that are homotopically nontrivial form barriers to transport, i.e. points on one side cannot cross to the other side. (We will abbreviate homotopically nontrivial invariant circles to just invariant circles.) When the perturbation increases, some of the circles cease to exist. The break-up of these circles (in both twist and nontwist maps) has been studied extensively.

In contrast to twist maps, the orbits of the standard nontwist map are generally found in pairs - two or none-for any winding number for any symmetry line. Also, for the standard nontwist map, the rotation numbers are bounded from above. The orbit with the extremal winding number occurs in the region in phase space where the twist condition fails. The curve in parameter space along which there is only one orbit of winding number $\omega$ is called the $\omega$-bifurcation curve and the orbit itself is called shearless. The rotation number of the shearless orbit is an extremum of all the rotation numbers of orbits in the map.

Remark 3. For the integrable map $b=0$, the circle with rotation number $a$ is shearless. Thus, the parameter values $(a, 0)$ belong to the $a$-bifurcation curve (i.e. to the $\omega$-bifurcation curve for $\omega=a$ ). It was shown in [17] that if $a$ is Diophantine, then there is an analytic $a$-bifurcation curve for small perturbations.

Shearless invariant circles are present in the phase space region where the twist condition fails. The break-up of shearless circles with noble winding numbers (with a continued fraction expansion ending in 1) has been studied extensively in [15,18,19,21]. The break-up of shearless invariant circles for nontwist time periodic Hamiltonian flows has been analysed in [20,25].

The critical circles for the three winding numbers

$$
\begin{aligned}
& \omega^{(1)}=[0,1,1,1, \ldots]=\frac{1}{\gamma}, \\
& \omega^{(2)}=[0,2,1,1,1, \ldots]=\frac{1}{\gamma^{2}}, \\
& \omega^{(3)}=[0,2,2,1,1,1, \ldots]=\frac{\gamma^{2}}{1+2 \gamma^{2}},
\end{aligned}
$$

where $\gamma$ is the golden mean, were studied in $[15,18,19,21]$. Here $\omega=\left[a_{0}, a_{1}, a_{2}, \ldots\right]$ denotes the continued fraction expansion [26] which is defined by

$$
\omega=\left[a_{0}, a_{1}, a_{2}, \ldots\right]:=a_{0}+\frac{1}{a_{1}+\left(1 /\left(a_{2}+\cdots\right)\right)} .
$$



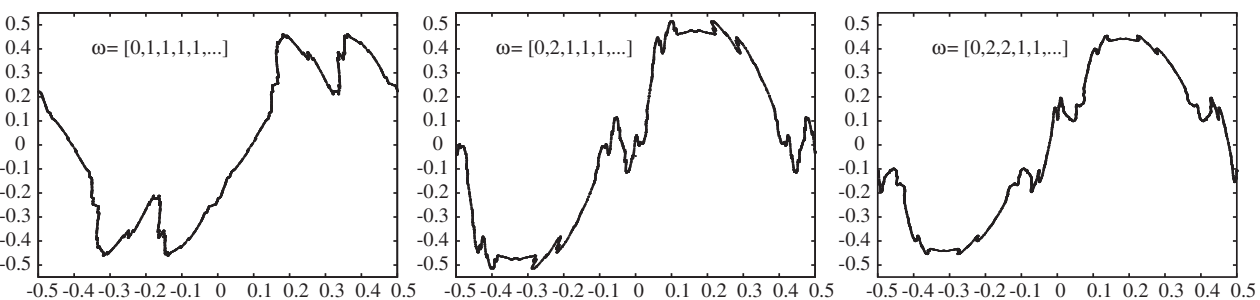

Figure 1. Critical circles with winding numbers $\omega^{(1)}, \omega^{(2)}$ and $\omega^{(3)}$ of (4).

The parameter values for which there is a critical circle were found in $[15,18,19,21]$ using Greene's residue criterion [27]. This method uses the observation that the invariant circle can be approximated by periodic orbits of winding numbers equal to the convergents obtained by truncating the continued fraction expansion. The residue criterion relates the stability of these periodic orbits to the break-up (loss of stability) of the invariant circle. A rigorous partial justification of this procedure for nontwist maps is presented in [17].

In the case of the standard nontwist map, the $\omega$-bifurcation curve for irrational $\omega$ is approximated by the numerically obtained limit of bifurcation curves of the periodic orbits. This limit shows a scaling behaviour, which further increases the accuracy of these calculations, for details of which we refer to [21]. The parameter values for which the above circles are critical are as follows:

$$
\begin{aligned}
& \left(a_{c}, b_{c}\right)^{(1)}=(0.686049108,0.74249354915518), \\
& \left(a_{c}, b_{c}\right)^{(2)}=(0.425160543,0.92446364703553), \\
& \left(a_{c}, b_{c}\right)^{(3)}=(0.45297741955,0.84582913999445) .
\end{aligned}
$$

These critical circles, which are shown in figure 1, are not smooth, and they show a selfsimilar structure $[15,18,19,21]$. Our aim here is to study numerically the smoothness of these critical circles and details of their fine scale structure using Fourier analysis. We will first describe the method used to parametrize invariant circles of nontwist maps.

\section{The parametrization function}

In contrast to the situation for twist maps, homotopically non-trivial invariant circles for nontwist maps can fail to be graphs. Indeed, our computations show that the critical shearless invariant circles are not graphs in any smooth coordinate system. A convenient way to describe circles that are not graphs is to find a parametric representation. In our case, it is convenient to choose this parametrization in a way that, moreover, turns the dynamics into a rotation. Such parametrization functions are often called 'hull functions', and they have been used extensively for the computation of periodic orbits and invariant circles. For example, they have been used as the basis for KAM theory in [28].

Therefore, the main object of this study will be a continuous map $K: \mathbb{T}^{1} \rightarrow \mathbb{T}^{1} \times \mathbb{R}$ whose range is the invariant circle we are seeking to describe and such that

$$
F \circ K(\theta)=K(\theta+\omega) \text {. }
$$

We will denote the components of $K$ by $K_{x}: \mathbb{T}^{1} \rightarrow \mathbb{T}^{1}$ and $K_{y}: \mathbb{T}^{1} \rightarrow \mathbb{R}$.

If we set $K(0)$ to be a point on the circle, applying (7) we obtain

$$
F^{n}(K(0))=K(n \omega) .
$$

Hence, it is very easy to compute numerically the function $K$ at points in the set $\{\theta=n \omega\}_{n=0}^{\infty}$. Since we are assuming that $\omega \notin \mathbb{Q}$, this is a dense set on the circle and, therefore, the continuous 

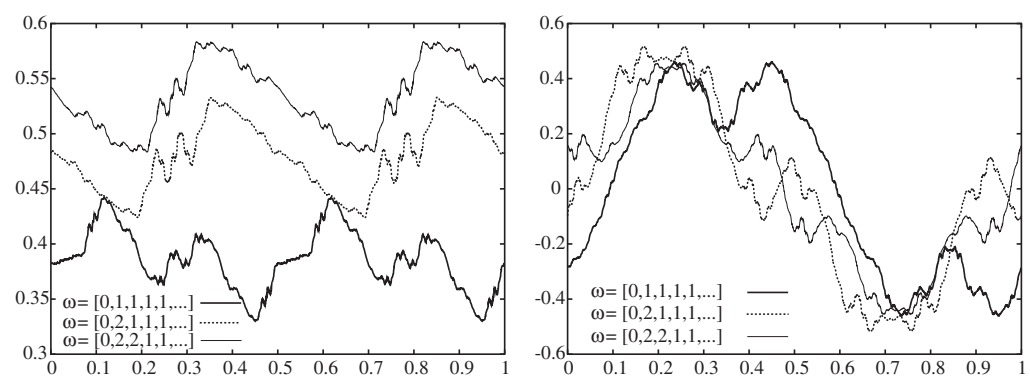

Figure 2. The functions $\tilde{K}_{x}(\theta)-\theta$ (left) and $\tilde{K}_{y}(\theta)$ (right) for the three winding numbers from (4). The symmetry properties (lemma 1 ) can be seen very clearly. The graphs of $\tilde{K}_{x}(\theta)-\theta$ have been shifted by a constant for clarity.

function $K$ is determined. For numerical applications, it is quite fortunate that $\{n \omega\}_{n=0}^{N}$ is very uniformly distributed in the torus.

The argument above shows that the continuous solutions of (7) are unique once we fix a point. More precisely, if $K$ and $\hat{K}$ solve (7), then there is a $\sigma$ such that

$$
\hat{K}(\theta)=K(\theta+\sigma) \text {. }
$$

Indeed, since $\hat{K}(0)$ is in the circle, it can be written as $K(\sigma)$. Then, applying (7), we obtain $\hat{K}(n \omega)=K(n \omega+\sigma)$. Hence, $(9)$ holds for a dense set.

We will also find it convenient to study lifts $\tilde{F}$ of the map $F$ and lifts $\tilde{K}$ of the function $K$. In general, a map $\tilde{K}: \mathbb{R} \rightarrow \mathbb{R} \times \mathbb{R}$ is a lift of a map $K: \mathbb{T}^{1} \rightarrow \mathbb{T}^{1} \times \mathbb{R}$ when the map $\tilde{K}$ becomes the map $K$ upon identifying the angle variables (the independent variable and the first coordinate of the range) that differ by an integer.

Of course, a lift corresponds to only one map $K$, but a map $K$ has infinitely many lifts. If $\tilde{K}$ is a lift of $K$, all the other lifts are given by $\tilde{K}(\theta+\ell)+(n, 0)$ where $\ell, n \in \mathbb{Z}$.

The lifts of a map $K$ in the annulus satisfy

$$
\tilde{K}(\theta+1)=\tilde{K}(\theta)+(n, 0) .
$$

The number $n$ measures how many turns are experienced by the angle variable in the range of $\tilde{K}$ when the angle variable in the domain of $\tilde{K}$ experiences one turn. We will call $n$ the index of the map $K$ or the index of the circle. In our case, we will consider only circles of index 1 . It is easy to see that circles of index larger than 1 have self-intersections, and circles of index 0 are homotopically trivial. The lift $\tilde{F}$ of our map $F$ of the annulus also satisfies

$$
\tilde{F}(x+1, y)=\tilde{F}(x, y)+(1,0) .
$$

Note that a consequence of $(10)$ is that $\tilde{K}_{x}(\theta)-\theta$ and $\tilde{K}_{y}(\theta)$ are periodic functions. These are the functions we will be studying numerically. This is equivalent to studying $K$ because if $K$ satisfies (7), then

$$
\tilde{F} \circ \tilde{K}(\tilde{\theta})=\tilde{K}(\tilde{\theta}+\omega) .
$$

The functions $\tilde{K}_{x}(\theta)-\theta$ and $\tilde{K}_{y}(\theta)$ for the shearless circles with winding numbers $\omega^{(1)}, \omega^{(2)}$, and $\omega^{(3)}$ are shown in figure 2.

\subsection{Symmetry properties}

The standard nontwist map (1) is symmetric under $S$ :

$$
F \circ S=S \circ F,
$$


where $S$ is the map of the annulus defined by

$$
S(x, y)=\left(x+\frac{1}{2},-y\right) \text {. }
$$

Note that $S$ is an involution:

$$
S^{2}=\mathrm{Id} \text {. }
$$

This symmetry implies the following properties for the functions $K$ solving (7).

Lemma 1. If the map $F$ satisfies (12) and the shearless circle is of index 1 , then $K$ satisfies

$$
S \circ K(\theta)=K\left(\theta+\frac{1}{2}\right),
$$

and any lift $\tilde{K}$ satisfies

$$
\tilde{K}_{x}\left(\theta+\frac{1}{2}\right)=\tilde{K}_{x}(\theta)+\frac{1}{2}, \quad \tilde{K}_{y}\left(\theta+\frac{1}{2}\right)=-\tilde{K}_{y}(\theta) .
$$

In particular $\tilde{K}_{x}(\theta)-\theta$ is of period $\frac{1}{2}$.

Proof. We have

$$
\begin{aligned}
F \circ S \circ K(\theta) & =S \circ F \circ K(\theta) \\
& =S \circ K(\theta+\omega),
\end{aligned}
$$

so that $S \circ K$ is also a solution of (7) and parametrizes the same circle. Hence, by the uniqueness result in 9 , there exists a number $\sigma$ for which

$$
S \circ K(\theta)=K(\theta+\sigma) .
$$

Noting that $S^{2}=\mathrm{Id}$, we conclude that

$$
K(\theta+2 \sigma)=K(\theta) .
$$

If $\sigma$ were an irrational number, then (15) would imply that $K(\theta)$ is constant, which is impossible. Hence, $\sigma$ is rational.

If $\sigma=n / m$ with $m$ odd, then

$$
S K(\theta)=S^{m} K(\theta)=K(\theta+n)=K(\theta) .
$$

This implies that $-K_{y}(\theta)=K_{y}(\theta)$, so that the circle is the $x$-axis, and that $K_{x}(\theta)+\frac{1}{2}=K_{x}(\theta)$, both of which are absurd.

Hence, the only possibility is $\sigma=n / 2 k$ with $n, k \in \mathbb{N}, \operatorname{gcd}(n, k)=1$ and, in particular $n$ is odd. Then, we can find $L, p \in \mathbb{N}$ such that $n L-p k=1$. Hence, iterating (15) $L$ times, we obtain

$$
K(\theta)=S^{2 L} K(\theta)=K\left(\theta+\frac{n L}{k}\right)=K\left(\theta+\frac{1}{k}\right) .
$$

Going to the lift we have that $\tilde{K}(\theta+1 / k)=\tilde{K}(\theta)+(i, 0)$, where $i \in \mathbb{Z}$. Applying this $k$ times, we obtain $\tilde{K}(\theta+1)=\tilde{K}(\theta)+(k i, 0)$. Since the index is $1, i \cdot k=1$ and therefore, $i=1$, $k=1$ and the result is proved.

\subsection{Reversibility}

The map (1) is reversible, which implies antisymmetry properties of the parametrization function. We follow the notation of [15]. 
Reversibility means that

$$
I_{0} \circ F=F^{-1} \circ I_{0}, \quad I_{0}^{2}=\mathrm{Id},
$$

where $I_{0}$ is a map of the annulus. Then it is easy to see that $I_{1}=F \circ I_{0}$ also satisfies

$$
I_{1} \circ F=F^{-1} \circ I_{1}, \quad I_{1}^{2}=\mathrm{Id} .
$$

The map $F$ is just a composition of these two involutions:

$$
F=I_{1} \circ I_{0} .
$$

For the map (1), we can take

$$
I_{0}(x, y)=(-x, y-b \sin (2 \pi x)), \quad I_{1}(x, y)=\left(-x+a\left(1-y^{2}\right), y\right) .
$$

Now, we investigate the implications of reversibility for the function $K$. If $K$ satisfies (7), we also have $F^{-1} \circ K(\theta)=K(\theta-\omega)$ or, denoting $L(\theta)=K(-\theta)$ we have

$$
F^{-1} \circ L(\theta)=L(\theta+\omega) \text {. }
$$

Also, composing (7) with $I_{0}$ and using (16), we get

$$
I_{0} \circ K(\theta+\omega)=F^{-1} \circ I_{0} \circ K(\theta) .
$$

Thus, $I_{0} \circ K$ and $L$ satisfy the same equation, and the argument leading to (9) implies that

$$
I_{0} \circ K(\theta)=L(\theta+\sigma)=K(-\theta-\sigma)
$$

for some $\sigma$. Clearly, $\sigma$ depends on $I_{0}$ and on the origin of coordinates we have chosen for $\theta$.

If we change the origin of coordinates in $\theta$ by $\sigma / 2$, we obtain that

$$
I_{0} \circ K(\theta)=K(-\theta) \text {. }
$$

Such $K$ will be said to be antisymmetric with respect to $I_{0}$. Note that if (20) holds, then $I_{0} \circ K(0)=K(0)$, so that the antisymmetry is evident if we choose $K(0)$ on the symmetry line (fixed point set) of $I_{0}$.

Composing (20) with $I_{1}$, we obtain

$$
\begin{aligned}
K(\theta+\omega) & =F \circ K(\theta)=I_{1} \circ I_{0} \circ K(\theta) \\
& =I_{1} \circ K(-\theta) .
\end{aligned}
$$

So, we see that if $K$ is antisymmetric with respect to $I_{0}$, a translation in $\theta$ by $\omega / 2$ will make it antisymmetric with respect to $I_{1}$. In this case, $K(0)$ belongs to the symmetry line for $I_{1}$.

For the case of the standard nontwist map, we obtain, using (21) for the $x$ component and (20) for the $y$ component, that

$$
\begin{aligned}
& K_{x}(-\theta)=-K_{x}(\theta), \\
& K_{y}(-\theta)=K_{y}(\theta+\omega) .
\end{aligned}
$$

Of course, the other two identities that we obtain from (20) and (21) are equivalent to (22) and (7). One consequence of (22) is that the Fourier series of $K_{x}(\theta)-\theta$ is simply a sine series or equivalently, the phases of the complex Fourier transform (which is what we implemented numerically) are either $\pi / 2$ or $3 \pi / 2$.

Equations (22) refer to parametrizations with $K(0)$ on a symmetry line. In our calculations, we have chosen to take $K(0)$ to be an indicator point [29], which is known for sure to belong to the shearless curve. In these coordinates, we can see that the phases of the Fourier coefficients should be $|k| \sigma+r_{k}$ where $r_{k}$ is either $\pi / 2$ or $3 \pi / 2$. This provides an independent check of the calculations of the Fourier coefficients and we discuss it in detail in section 5.1.

We could use (14) to compute only the even (respectively odd) Fourier coefficients of $K_{x}(\theta)-\theta$ (respectively $K_{y}(\theta)$ ) and carry out the analysis only for them. We have chosen not to hardwire this symmetry into the calculations of Fourier spectra. In this way, the size of the odd (respectively even) coefficients gives us an estimate of the numerical error in our computations. 


\section{Computing the regularity of the parametrization functions}

In this section, we will briefly describe the methods we used to compute the regularity of the parametrization functions. A much more extensive discussion can be found in [22], to which we refer for further details and for references to the mathematical literature.

In this paper, we will only use the two methods that were found to be the most accurate in [22], namely the so-called 'continuous Littlewood-Paley' and the 'difference' methods. Notably, we do not consider the methods based on wavelets that were implemented in [22]. We refer to [22] for a comparison of various numerical methods.

\subsection{Some mathematical results}

Following the standard mathematical notation, we will express the regularity of the functions considered by saying that the function belongs to certain spaces in a family indexed by a number. We will use the following definition.

Definition 1. For $\alpha=n+\beta$ with $n \in \mathbb{Z}, \beta \in(0,1)$, we say that the function $K$ is in $\Lambda_{\alpha}(\mathbb{T})$ when $K$ is $n$ times differentiable and

$$
\left|D^{n} K(\theta)-D^{n} K(\tilde{\theta})\right| \leqslant C|\theta-\tilde{\theta}|^{\beta}
$$

for some constant $C$.

What we mean by the regularity of a function is the largest value of $\alpha$ so that the function belongs to $\Lambda_{\alpha}$.

The integer part of $\alpha$ is the number of derivatives and the fractional part of $\alpha$ is the Holder exponent of the highest derivative.

The above definition does not cover the case when $\alpha$ is an integer. It is a fact of life that many of the characterizations we will use become delicate when $\alpha$ is an integer. Fortunately, for the applications here, we do not need to consider the case when $\alpha$ is an integer.

The following results can be found in [30, chapter 5, lemma 5] and [31], respectively.

Theorem 1 (CLP). The function $K$ is in $\Lambda_{\alpha}(\mathbb{T})$ if and only if for some $\eta \geqslant 0$ there exists a $C>0$, such that for any $t>0$

$$
\left\|\left(\frac{\partial}{\partial t}\right)^{\eta} \mathrm{e}^{-t \sqrt{-\Delta}} K\right\|_{L^{\infty}(\mathbb{T})} \leqslant C t^{\alpha-\eta} .
$$

Remark 4. If the inequality (24) holds for some $\eta \geqslant 0$, then it holds for all $\eta \geqslant 0$.

Remark 5. The operator $\mathrm{e}^{-t \sqrt{-\Delta}}$ is the Poisson kernel in the circle, which appears very often in the study of boundary value problems.

Theorem 2 (FD). Let $\mathcal{D}_{h}^{n}$ be the finite difference operator,

$$
\left(\mathcal{D}_{h}^{n} K\right)(\theta):=\sum_{j=0}^{n}(-1)^{j}\left(\begin{array}{l}
n \\
j
\end{array}\right) K(\theta+(n-2 j) h) .
$$

Then $K \in \Lambda_{\alpha}(\mathbb{T})$ if and only if $\exists C>0$ such that $\forall h \in \mathbb{T}$

$$
\left\|\mathcal{D}_{\theta}^{n} K\right\|_{L^{\infty}(\mathbb{T})} \leqslant C|h|^{\alpha} \text {. }
$$

Remark 6. The mathematical theory only requires (24) and (25) to be upper bounds. Nevertheless, we note that in our case, the bounds become identities for a significant range of parameters. The fact that the upper bounds are saturated is very possibly a consequence of the fact that, because of the renormalization picture, the functions we are considering are very 
self-similar. The same effect was also observed for the functions considered in [22] (see also the discussion in section 3.2).

\subsection{Some remarks on the numerical implementation}

Both numerical methods considered here are made easier if the function $K$ is specified at equally spaced points. The computation described in section 3 , however, gives the function values for $\{\theta=n \omega\}_{n=0}^{M}$. Hence, the first step is to compute the function on an evenly spaced grid of size $2^{-N}$. This is done using a cubic spline interpolation. Here, $N$ is chosen such that $2^{N} \approx M$.

To use theorem 2 numerically, we note that substituting $h=2^{-j}$ and $n=1$ in equation (25) gives

$$
\log _{2}\left\|\mathcal{D}_{2^{-j}}^{1} K\right\|_{L^{\infty}(\mathbb{T})} \leqslant \text { const }-\alpha j .
$$

Thus, the finite difference method simply evaluates the difference operators for various $j$, and we fit a straight line to a logarithmic plot of $\left\|\mathcal{D}_{2^{-j}}^{1} K\right\|_{L^{\infty}(\mathbb{T})}$ versus $j$.

For the CLP method (theorem 1), the most effective way to compute the Poisson kernel is to observe that it is diagonal in Fourier series. Specifically, if

$$
K(\theta)=\sum_{n \in \mathbb{Z}} \hat{K}_{n} \mathrm{e}^{-2 \pi i n \theta}
$$

then

$$
\left(\frac{\partial}{\partial t}\right)^{\eta} \mathrm{e}^{-t \sqrt{-\Delta}} K(\theta)=\sum_{n \in \mathbb{Z}}(-2 \pi|n|)^{\eta} \hat{K}_{n} \mathrm{e}^{-2 \pi t|n|} \mathrm{e}^{-2 \pi i n \theta} .
$$

Using the fast Fourier transform-which becomes possible once we have the function interpolated in an equally spaced grid - it is very efficient to compute the Fourier coefficients, multiply them by the appropriate coefficients, compute the inverse Fourier transform and then take the supremum over $\theta$. By repeating the computation for several values of $t$ and then fitting a straight line in a double logarithmic plot of $\left\|(\partial / \partial t)^{\eta} \mathrm{e}^{-t \sqrt{-\Delta}} K\right\|_{L^{\infty}(\mathbb{T})}$ versus $t$, we obtain a good estimation of the regularity.

In the application of the CLP method, it is convenient to choose $\eta$ in such a way that the power in $t$ that results in the decay is as close to zero as possible. The reason is that the coefficients that are very close to zero are strongly affected by round-off error in the calculations.

Remark 7. In [22] it was argued that the CLP method was numerically more stable while the difference method was more susceptible to numerical error. Hence, in this paper, we will obtain the regularities from the CLP method and use the FD method as a confirmation.

\section{Results and discussion}

\subsection{Visual observations of the Fourier spectrum}

As mentioned earlier, the critical circles have a self-similar structure which is manifest in the self-similarity of the parametrization functions. We can see this by looking at their Fourier spectra (the modulus of the Fourier coefficients). Figure 3 shows the spectra of $K_{x}(\theta)-\theta$ for the three frequencies studied. We see that the Fourier spectra are periodic with a period $6 \log _{10} \gamma$. A similar structure is also observed for the spectra of $K_{y}(\theta)$. We note that the peaks in the spectra are observed at values that are the denominators of the truncations 

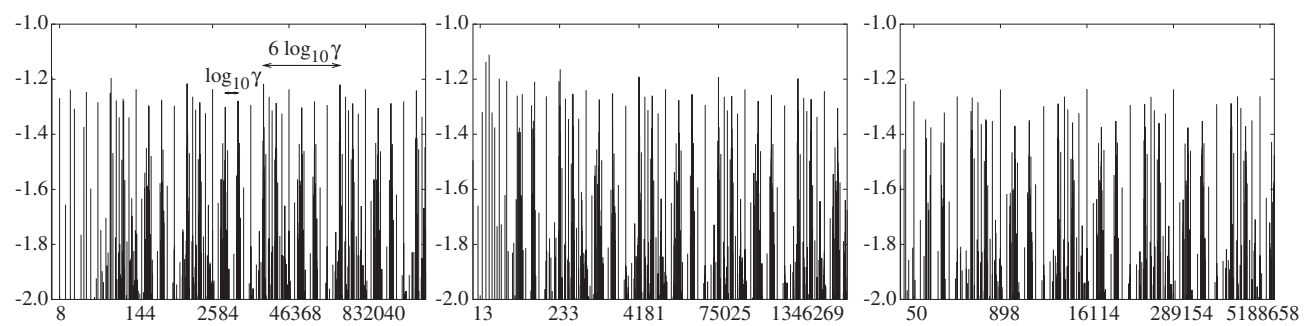

Figure 3. Plot of $\log _{10}\left(n\left|\hat{K}_{n}\right|\right)$ versus $\log _{10} n$ where $\hat{K}_{n}$ are the Fourier coefficients of $K_{x}(\theta)-\theta$ for $\omega^{(1)}$ (left), $\omega^{(2)}$ (centre) and $\omega^{(3)}$ (right). The abscissas marked here are the denominators of continued fraction convergents for that specific frequency.
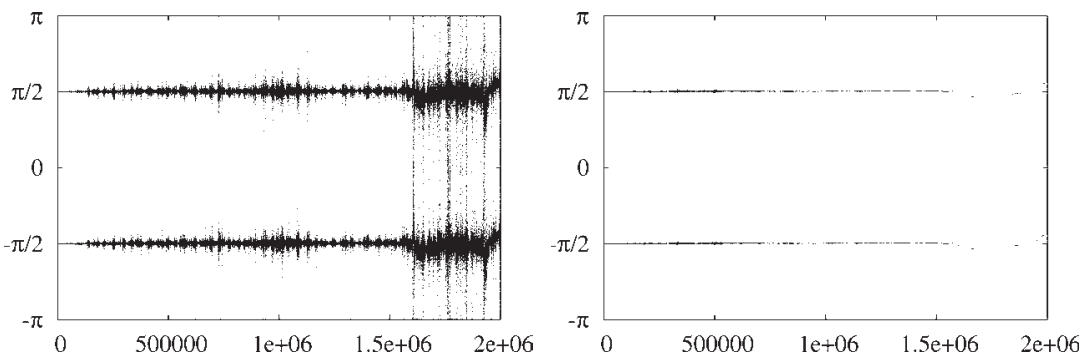

Figure 4. The phases of the Fourier transform of $K_{x}(\theta)-\theta$ with $K_{x}(0)$ chosen to be on the symmetry line of $I_{0}$. Left panel shows all the phases while the right panel shows phases for $n$ for which $\log _{10} \hat{K}_{n}>-9.5$.

of the continued fraction expansion of the corresponding winding number, with every sixth denominator showing a major peak while those in between show minor peaks. Specifically, the peaks occur at Fibonacci numbers only for the first two winding numbers.

Remark 8. In the case of twist maps, the period of such a plot is $\log _{10} \gamma$. The factor of 6 for the nontwist maps is related to the fact that the critical nontwist map belongs to a period-12 cycle of the renormalization group operator whereas the critical twist map is a fixed point of that operator.

As we noted in section 3.2, the functions $K_{x}(\theta)-\theta$ and $K_{y}(\theta)$ can be made antisymmetric with an appropriate choice of initial conditions, i.e. values of these functions at $\theta=0$. These initial conditions (the points of intersection of the critical shearless circle with the symmetry lines) are known with a limited accuracy whereas the indicator points that we used as initial conditions are guaranteed to lie on the shearless circle. Indeed, in our calculations, the odd (respectively even) Fourier coefficients of $K_{x}(\theta)-\theta$ (respectively $K_{y}(\theta)$ ) are smaller when using the indicator points $\left(10^{-11}\right.$ to $\left.10^{-10}\right)$ than when using the symmetry lines $\left(10^{-10}\right.$ to $\left.10^{-9}\right)$.

Since we chose the indicator point as initial conditions, the phases of Fourier spectra lie on straight lines. The slope of these lines is $2 \pi y_{0}$ where $y_{0}$ is the point of intersection of the shearless circle with the symmetry line of $I_{0}$ while the intercept is $\pi / 2$ or $3 \pi / 2$.

To check the numerical accuracy of the Fourier coefficients, we also calculated the spectra after choosing the initial conditions to make the function $K_{x}(\theta)-\theta$ antisymmetric, i.e. choosing $K_{x}(0)$ to be a fixed point of $I_{0}$. Figure 4 shows the phases for all $n<2 \times 10^{6}$ (left panel) and also for $n$ for which $\log _{10}\left|\hat{K}_{n}\right|>-9.5$ (right panel). We see that the phases are indeed $\pi / 2$ or $3 \pi / 2$ for all $n<1.6 \times 10^{6}$ with $\log _{10}\left|\hat{K}_{n}\right|>-9.5$. 


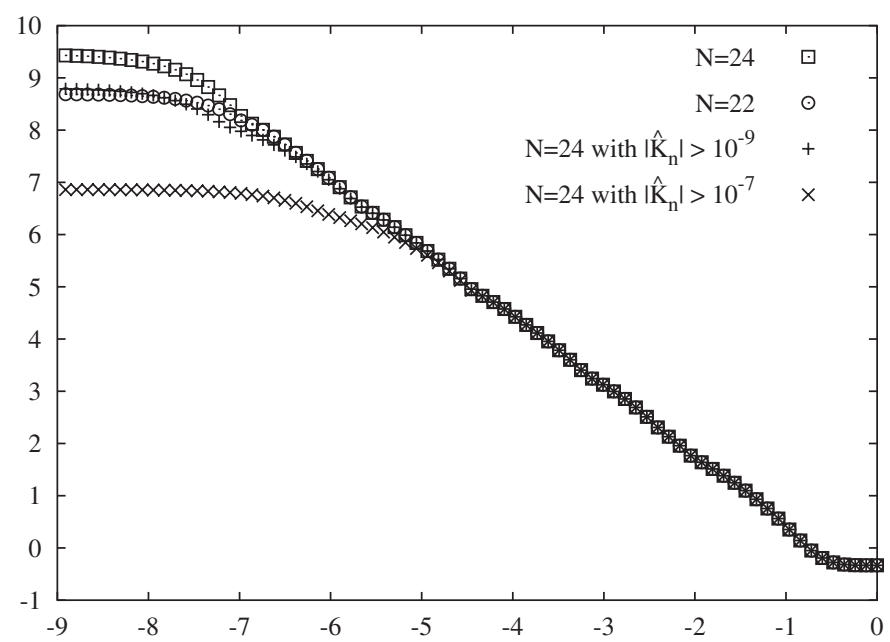

Figure 5. Comparing the CLP method for different grid sizes $2^{-N}$ for $N=24(\square)$ and $N=22(\odot)$; we also show the results from the runs with Fourier coefficients of magnitude less than $10^{-9}(+)$ or $10^{-7}(\times)$ set to zero.
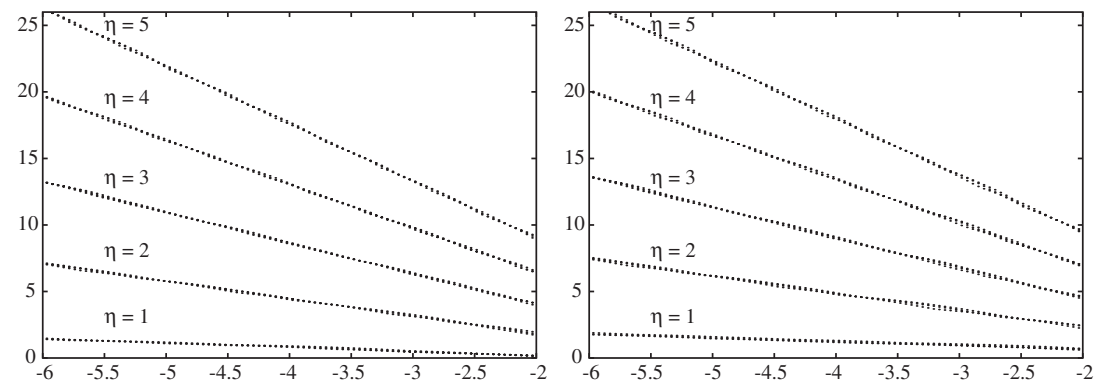

Figure 6. The plots of $\log _{10}\left\|(\partial / \partial t)^{\eta} \mathrm{e}^{-t \sqrt{-\Delta}}(\tilde{K}(\theta)-\theta)\right\|_{L^{\infty}(\mathbb{T})}$ versus $\log _{10} t$ for $K_{x}(\theta)-\theta$ (left) and $K_{y}(\theta)$ (right) used to calculate the regularity using the CLP method.

\subsection{Regularity using the CLP method}

We compared results from different grid sizes to check for robustness of the methods used here. Figure 5 shows the plots of $\log _{10}\left\|(\partial / \partial t)^{2} \mathrm{e}^{-t \sqrt{-\Delta}}(\tilde{K}(\theta)-\theta)\right\|_{L^{\infty}(\mathbb{T})}$ versus $\log _{10} t$ computed with $2^{-N}$ number of points for $N=24(\odot)$ and $N=22(\odot)$. In order to assess the accuracy, we also calculated the above norms for $N=24$ by setting all the Fourier coefficients $\hat{K}_{n}$ with magnitude less than $10^{-7}$ or $10^{-9}$ to zero, shown in figure 5 with $\times$ and + , respectively. We see that the plot for $N=22$ compares very well with that for $N=24$ with all $\left|\hat{K}_{n}\right|<10^{-9}$ set to zero. We also see that for $\log _{10} t>-5$, all the calculations give essentially the same results.

The observations in figure 5 make it apparent that there is a substantial range of values of $\log _{10} t$ for which (24) is close to an equality (see also figure 6.) This range is limited on one side (of small $\log _{10} t$ ) by the numerical error (round-off and truncation of Fourier series) and on the other side (of $\operatorname{larger} \log _{10} t$ ) by the universal self-similar structure, which is only true asymptotically.

Once we have identified the range where (24) is an equality, a good measure of the regularity is obtained by the slope of the linear fit within that range. We will see in section 5.3 
Table 1. Regularities of the parametrization functions for three frequencies using the CLP method with different $\eta$. The error shown is the standard error from the linear regression. The ranges of $\log _{10} t$ were chosen so that corrections to the linear behaviour are periodic, as explained in detail in section 5.3.

\begin{tabular}{lllll}
\hline & $\eta$ & $\omega^{(1)}$ & $\omega^{(2)}$ & $\omega^{(3)}$ \\
\hline$K_{x}(\theta)-\theta$ & 1 & $0.700 \pm 0.005$ & $0.718 \pm 0.004$ & $0.698 \pm 0.005$ \\
& 2 & $0.679 \pm 0.006$ & $0.656 \pm 0.005$ & $0.671 \pm 0.005$ \\
& 3 & $0.647 \pm 0.018$ & $0.647 \pm 0.018$ & $0.636 \pm 0.015$ \\
$K_{y}(\theta)$ & 4 & $0.687 \pm 0.007$ & $0.669 \pm 0.007$ & $0.676 \pm 0.007$ \\
& 5 & $0.683 \pm 0.008$ & $0.667 \pm 0.009$ & $0.672 \pm 0.009$ \\
& 1 & $0.724 \pm 0.007$ & $0.728 \pm 0.003$ & $0.730 \pm 0.006$ \\
& 2 & $0.700 \pm 0.006$ & $0.692 \pm 0.006$ & $0.692 \pm 0.006$ \\
& 3 & $0.645 \pm 0.015$ & $0.622 \pm 0.015$ & $0.629 \pm 0.015$ \\
& 4 & $0.690 \pm 0.008$ & $0.675 \pm 0.008$ & $0.680 \pm 0.008$ \\
& 5 & $0.690 \pm 0.005$ & $0.702 \pm 0.006$ & $0.698 \pm 0.005$ \\
\hline
\end{tabular}

Table 2. Regularities of $K_{x}(\theta)-\theta$ and $K_{y}(\theta)$ for the three frequencies.

\begin{tabular}{llll}
\hline & $\omega^{(1)}$ & $\omega^{(2)}$ & $\omega^{(3)}$ \\
\hline$K_{x}(\theta)-\theta$ & $0.68 \pm 0.02$ & $0.67 \pm 0.02$ & $0.67 \pm 0.01$ \\
$K_{y}(\theta)$ & $0.69 \pm 0.02$ & $0.68 \pm 0.03$ & $0.69 \pm 0.03$ \\
\hline
\end{tabular}
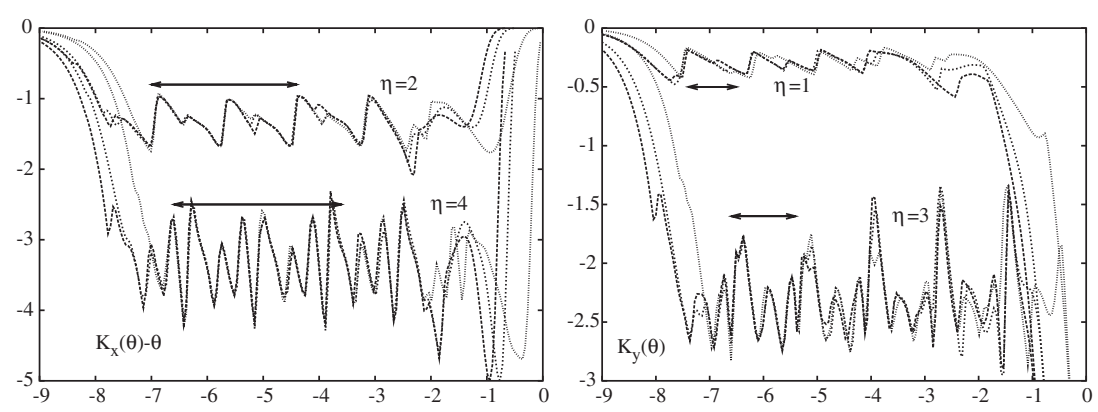

Figure 7. The finite differences in the plots of $\log _{10}\left\|(\partial / \partial t)^{2} \mathrm{e}^{-t \sqrt{-\Delta}} K\right\|_{L^{\infty}(\mathbb{T})}$ versus $\log _{10} t$ for $\eta=2,4$ for $K_{x}(\theta)-\theta$ (left) and for $\eta=1,3$ for $K_{y}(\theta)$ (right). The ranges selected for getting

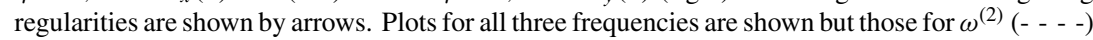
and $\omega^{(3)}(-\ldots)$ have been shifted horizontally to match that of $\omega^{(1)}(\cdots)$.

that the deviations from the linear fit show a universal behaviour. Thus, a systematic way of choosing the range of fit is where the universality applies. Table 1 shows the regularities found using such ranges. The errors shown are the standard deviations of the linear regression.

Increasing the number of values of $t$ at which the norm is calculated obviously decreases the standard deviation of the linear fit. Thus, the errors shown in table 2 can be made smaller or larger. Of course, choosing different ranges of $\log _{10} t$ for the linear fit give slightly different regularities, depending on whether the jumps in the deviation from the linear behaviour (as seen in, e.g., figure 7) are included or not. Thus, the errors shown in table 2 include these systematic errors from all five values of $\eta$ used here.

Remark 9. The three frequencies considered here are all noble numbers. As a consequence, the invariant circles with rotation numbers $\omega^{(2)}$ and $\omega^{(3)}$ for the standard nontwist map are equivalent to circles with rotation number $\omega^{(1)}$ for maps which are obtained from the standard 
nontwist map by renormalization. The fact that the regularities we find here are the same for all the circles is equivalent to saying that they are the same for circles of rotation number $\omega^{(1)}$ for different maps. Hence, the fact that the regularities for different frequencies are seen to be the same is an evidence of universality of critical noble invariant circles.

\subsection{Universal corrections to the CLP bound}

If the inequalities (24) were identically saturated, we would have that $R\left(\log _{10} t\right) \equiv$ $\log _{10}\left\|(\partial / \partial t)^{2} \mathrm{e}^{-t \sqrt{-\Delta}} K\right\|_{L^{\infty}(\mathbb{T})}$ would be an affine function of $\log _{10} t$ and the plot of $R(l)$ versus $l$ would be a straight line.

Nevertheless, it is a prediction of renormalization group theory that there are systematic deviations from the linear behaviour of $R$. In particular, there should be a periodic oscillation.

As was argued in [22], the deviation of $R(l)$ from an affine behaviour is related to the fact that the scaling predicted by the renormalization group is not a continuous scaling but just a discrete one. The renormalization group predicts that, for sufficiently small $t>0$ (i.e. large negative $\left.\log _{10} t\right)$, there are numbers $a, b$ such that $R(l+a) \approx R(l)+b$. This leaves open the possibility that, superimposed on the linear behaviour of $R(l)$ versus $l$, there is a periodic behaviour. That is, $R(l) \approx(b / a) l+P(l)$ where $P(l)$ is a function of period $a$. According to the renormalization group picture, the function $P$ should be universal. Of course, for different functions in the same universality class, the asymptotic behaviour may manifest itself only for very large negative values of $l$.

The periodic correction to $R(l)$ is closely related to the approximate periodicity found for the Fourier coefficients $\left|\hat{K}_{n}\right|$ as a function of $\log _{10}|n|$, which we already observed in figure 3 . The universality of the periodic function $P(l)$ is related to the fact that there is an asymptotic shape for the period of the scaled Fourier coefficients.

We have verified the existence of a universal periodic function $P(l)$ by computing the finite differences in the plot of $R(l)$ versus $l$. The results for $\eta=2,4$ for $K_{x}(\theta)-\theta$ and for $\eta=1,3$ for $K_{y}(\theta)$ are depicted in figure 7 . We also see that, as mentioned earlier, the smaller values of $\eta$ are more accurate, i.e. the periodicity of these corrections is more clearly seen for smaller $\eta$.

We note that the period of the oscillations in these plots is six times larger than the period observed in the problems in [22]. This is due to the fact that the renormalization operator in our case has period 6 rather than period 1 as in the cases considered in [22]. This effect is clearly visible in the period of the Fourier coefficients, as seen already in section 5.1.

We also note that for different $\omega$ we obtain a different phase in the periodic correction. This is due to the fact that our $\omega$ have a different entry in the first place of the continued fraction expansion, so that they need one more application of the renormalization to start exhibiting the periodic behaviour. Hence, in the figure, we have shifted the finite differences to exhibit that, for our examples, they agree as predicted by universality.

The existence of a periodic correction to the linear dependence of $R$, as well as its agreement in the examples studied, gives us good confidence that we are observing real effects. From a more practical point of view, it gives us the following criterion: we fit a straight line to the plot of $R(l)$ versus $l$ only in the region where the periodicity of the correction is manifest. These regions are shown in figure 7 . All these tasks were greatly facilitated by the remarkable public domain program xmgrace.

\subsection{Regularity using the finite difference method}

As with the CLP method, we first compare results for different grid sizes. The plot of $\log _{2}\left\|\mathcal{D}_{2^{-j}}^{1} K\right\|_{L^{\infty}(\mathbb{T})}$ versus $j$ is shown in figure 8 for $\tilde{K}_{x}(\theta)-\theta$ for the frequency $\omega^{(1)}=1 / \gamma$ 


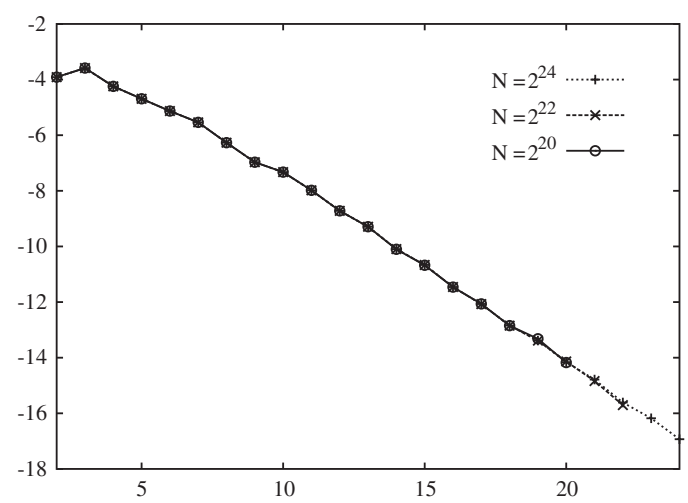

Figure 8. Comparing the plots of $\log _{2}\left\|\mathcal{D}_{2^{-j}}^{1} K\right\|_{L^{\infty}(\mathbb{T})}$ versus $j$ with $2^{N}$ points for $N=20,22,24$ for $\tilde{K}_{x}(\theta)-\theta$ for the frequency $\omega^{(1)}$.
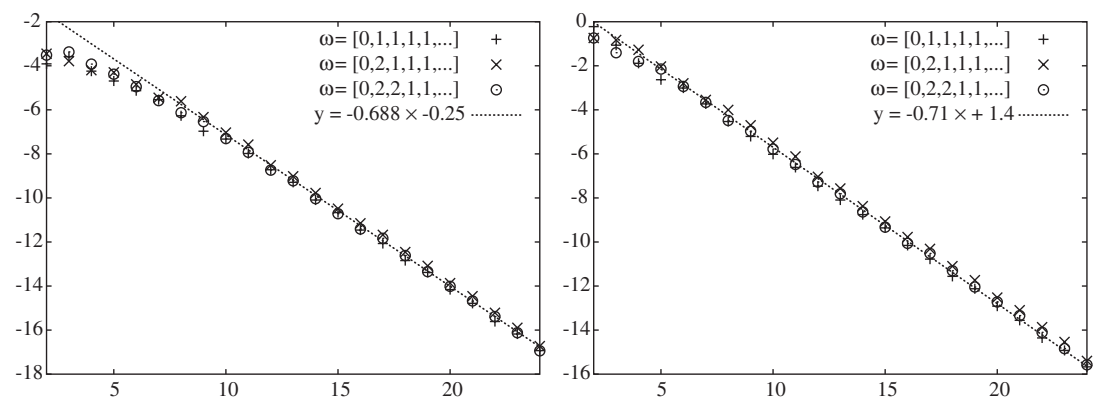

Figure 9. The plots of $\log _{2}\left\|\mathcal{D}_{2^{-j}}^{1} K\right\|_{L^{\infty}(\mathbb{T})}$ versus $j$ for $K_{x}(\theta)-\theta$ (left) and $K_{y}(\theta)$ (right) used to calculate the regularity using the finite difference method.

for $N=20,22$ and 24. We note that there is a break at $j=10$. The regularity obtained from the plot with $j>10$ agrees very well with the CLP method while that obtained from all the points with $j>5$ is lower. Since this method is affected more by round-off errors, we have used it only as a quick confirmation of results from the CLP method. The agreement between the two methods is much better for $K_{y}(\theta)$ probably because the round-off errors in iterating the map $F$ propagate faster along the angle coordinate $(x)$ (and hence in $K_{x}(\theta)$ ) than in the action coordinate $(y)$ (and hence in $\left.K_{y}(\theta)\right)$ [27, appendix C].

Figure 9 compares the plots of $\log _{2}\left\|\mathcal{D}_{2^{-j}}^{1} K\right\|_{L^{\infty}(\mathbb{T})}$ versus $j$ for $N=24$ for all the three frequencies for $K_{x}(\theta)-\theta$ and $K_{y}(\theta)$ with the line whose slope is the regularity obtained from the CLP method. We see that the bound in (25) is not saturated for $j<10$ for $K_{x}(\theta)-\theta$.

From the regularities of the parametrization functions $K_{x}(\theta)-\theta$ and $K_{y}(\theta)$ for the three frequencies $\omega^{(1)}, \omega^{(2)}$ and $\omega^{(3)}$ shown in table 2, we conclude that the universal regularity of $K(\theta)$ for critical shearless noble circles is $0.69 \pm 0.03$.

\section{Acknowledgments}

RL has been partially supported by NSF. NP has been partially supported by NSF grant DMS-0405903. AA has been partially supported by the US Department of Energy Contract no DE-FG01-96ER-54346. We thank the Mathematics Department at UT-Austin for the use of computer facilities. 


\section{References}

[1] Stix T H 1976 Phys. Rev. Lett. 3610

[2] Oda G A and Caldas I L 1995 Chaos Solitons Fractals 515

[3] Balescu R 1998 Phys. Rev. E 583781

[4] Horton W, Park H B, Kwon J M, Strozzi D, Morrison P J and Choi D I 1998 Phys. Plasmas 53910

[5] Ullmann K and Caldas I L 2000 Chaos Solitons Fractals 112129

[6] Del-Castillo-Negrete D and Firpo M-C 2002 Chaos 12496

[7] Munteanu A, Petrisor E, García-Berro E and José J 2003 Commun. Nonlinear Sci. Numer. Simul. 8355

[8] Petrisor E, Misguich J H and Constantinescu D 2003 Chaos Solitons Fractals 181085

[9] Del-Castillo-Negrete D and Morrison P J 1993 Phys. Fluids A 5948

[10] Howard J E and Humpherys J 1995 Physica D 80256

[11] Simó C 1998 Regul. Chaotic Dyn. 3180

[12] Voyatzis G, Meletlidou E and Ichtiaroglou S 2002 Chaos Solitons Fractals 141179

[13] Dullin H R and Meiss J D 2003 Chaos 131

[14] Franks J and Le Calvez P 2003 Ergod. Theory Dyn. Syst. 23111

[15] Del-Castillo-Negrete D, Greene J M and Morrison P J 1996 Physica D 911

[16] Del Castillo-Negrete D, Greene J M and Morrison P J 1997 Physica D 100311

[17] Delshams A and de la Llave R 2000 SIAM J. Math. Anal. 311235

[18] Wurm A 2002 Renormalization group applications in area-preserving nontwist maps and relativistic quantum field theory PhD Thesis The University of Texas at Austin

[19] Apte A, Wurm A and Morrison P J 2003 Chaos 13421

[20] Koch H and Gaidashev D 2004 Nonlinearity 171713

[21] Apte A 2004 Numerical studies of the standard nontwist map and a renormalization group framework for breakup of invariant tori PhD Thesis University of Texas at Austin

[22] de la Llave R and Petrov N P 2001 Exp. Math. 11219

[23] Petrov N 2002 Methods of dynamical systems, harmonic analysis and wavelets applied to several physical problems PhD Thesis University of Texas at Austin

[24] Apte A, Wurm A and Morrison P J 2005 Physica D 20047

[25] Gaidashev D 2003 Preprint mp_arc \# 03-542

[26] Khinchin A Y 1964 Continued Fractions 3rd edn (Chicago, IL: University of Chicago Press)

[27] Greene J M 1979 J. Math. Phys. 201183

[28] de la Llave R, Gonzalez A, Jorba A and Villanueva J 2005 Nonlinearity 18855

[29] Shinohara S and Aizawa Y 1998 Prog. Theor. Phys. 100219

[30] Stein E M 1970 Singular Integrals and Differentiability Properties of Functions (Princeton, NJ: Princeton University Press)

[31] Krantz S G 1983 Exposition. Math. 1193 